\title{
Children under 5 years are at risk for tuberculosis after occasional contact with highly contagious patients: outbreak from a smear-positive healthcare worker
}

To the Editor:

The World Health Organization (WHO), jointly with experts from tuberculosis (TB) low-incidence countries, developed a framework for TB elimination $[1,2]$.

While the traditional basis for TB control is rapid diagnosis and effective treatment of infectious TB patients, a crucial intervention for TB elimination is diagnosis and treatment of latent TB infection (LTBI) [3]. By reducing the pool of infected individuals at risk, the emergence of future cases of TB will be prevented. Among the core activities recommended by the WHO for TB low-incidence countries to reach $\mathrm{TB}$ elimination, protecting vulnerable populations and preventing transmission of $\mathrm{TB}$ infection in the community are of crucial importance $[2,3]$.

In general, the lower the TB incidence, the less are the experience and ability of clinicians to promptly and correctly diagnose and treat the disease [2]. As a consequence of delays in diagnosis, in low-incidence settings, TB micro-epidemics become relatively frequent [4-6]. Recently, several micro-epidemics have been described in low TB-incidence countries; some were generated by organisational pitfalls [4], others involved difficult-to-treat strains of Mycobacterium tuberculosis [5] or had healthcare workers as the index case [6]. In all of them the public health response started from assessment of contacts of the index case in concentric circles, according to the stone-in-the-pond principle [7] and assuming that at least $8 \mathrm{~h}$ of continuous contact are necessary to infect an individual $[8,9]$. However, the likelihood of TB infection transmission following short exposure $(<8 \mathrm{~h})$ of children to an infectious adult in a confined space is not fully known.

A recent micro-epidemic that occurred in Trieste (Northern Italy) may help us to understand the transmission dynamics of TB in adults and children better.

A female paediatrician (index case), responsible for vaccination of children $<6$ years of age, was diagnosed with highly infectious TB ( $>10$ bacilli/field in the sputum smear at the direct microscopic examination or $4+$, and large cavitary lesions, figure 1a). The patient reported with delay fatigue, productive coughing for 9 months and very low body weight (BMI 15.4). She never smoked. Prior to diagnosis, the index case was in occasional contact with children attending the clinic for vaccination purposes (session duration estimated at 10-20 min). Furthermore, the index case attended two further events for $<8 \mathrm{~h}$ before diagnosis: a refresher course ( 2 weeks before: contact with colleagues) and a wedding ( 3 days before: contact with invited guests, figure $1 \mathrm{~b}$ ).

We describe here an epidemic of TB among children of $<6$ years of age, and compare the relative risk of being infected (and the risk of developing TB disease) in children versus adults following an occasional contact with a highly infectious index case.

Given the potential infectiousness of the index case, an outbreak control committee with all relevant stakeholders was promptly established $[10,11]$.

@ERSpublications

Children under 5 years of age are at risk for tuberculosis after occasional contact with highly contagious patients http://ow.ly/RqAn30fKdGk

Cite this article as: Luzzati R, Migliori GB, Zignol M, et al. Children under 5 years are at risk for tuberculosis after occasional contact with highly contagious patients: outbreak from a smear-positive healthcare worker. Eur Respir J 2017; 50: 1701414 [https://doi.org/10.1183/13993003.01414-2017]. 


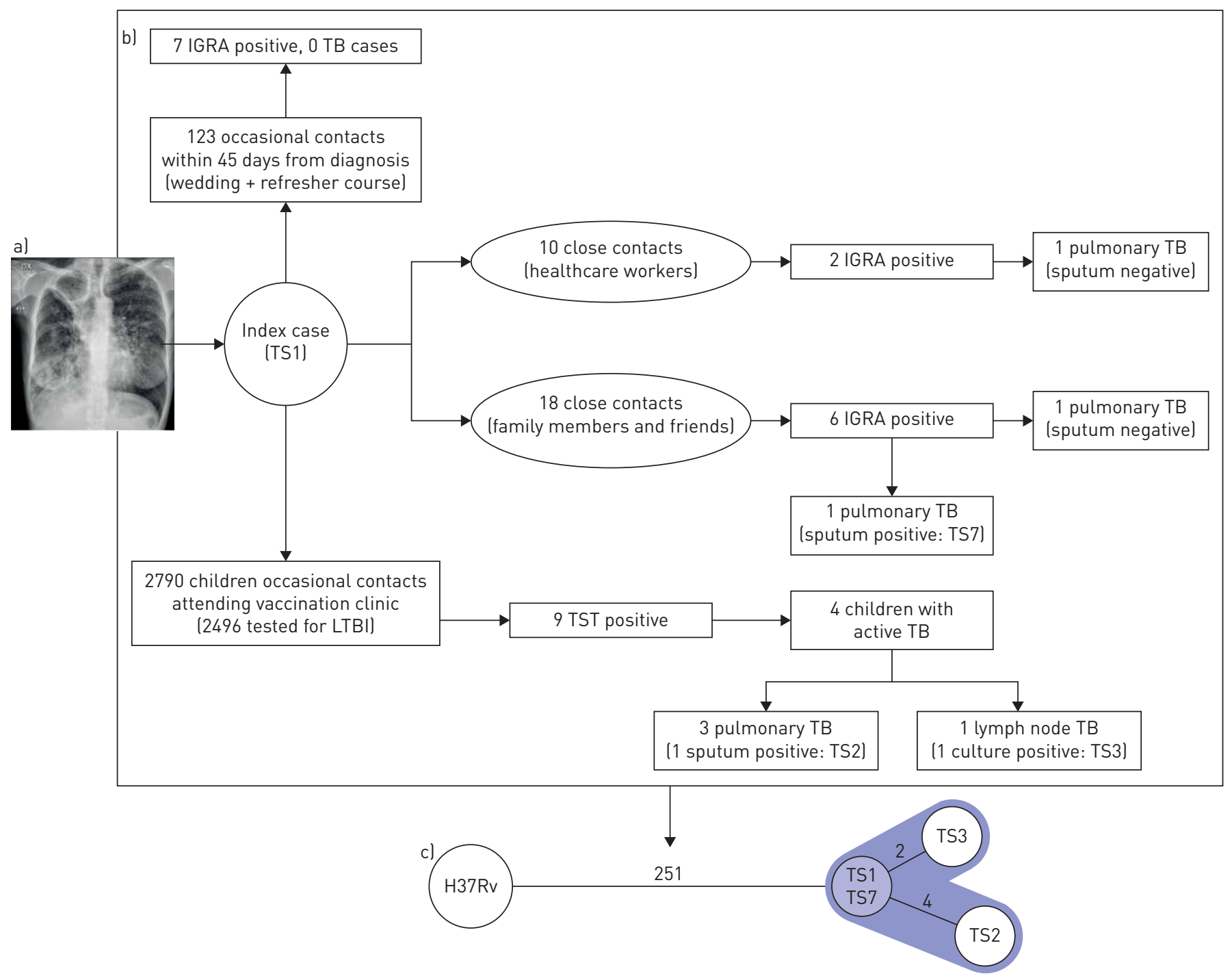

FIGURE 1 a) Chest radiography of the index patient, a 61-year-old female, at diagnosis, with evident cavities; the image is typical of a diseases which evolved over several months. b) Flow-chart of the contact investigation with latent tuberculosis (TB) and active TB patients. c) Epidemiological genomic analysis of the TB bacilli strains from index case (TS1) and secondary cases (TS2, TS3, TS7) showing 0-4 allelic variants in comparison with the index case. The index case shows a heterogeneous bacterial population with a majority (70\%) population responsible for transmission to TS7 and TS3 and a minority responsible for the transmission to TS2. The difference in number of alleles between this minority population in the index case and TS2 is reduced from four to two alleles. Strains were classified as EuroAmerican superlineages (spoligo 775 and MIRU-VNTR orphan). Genomes of reference strain H37RV is reported in the figure. IGRA: Interferon- $\gamma$ release assay; LTBI: latent TB infection; TST: tuberculin skin test.

The contact investigation included all close contacts of the index case, every child $<6$ years of age vaccinated by the paediatrician in the 12 months prior to diagnosis and occasional contacts for $<8 \mathrm{~h}$ in the previous 45 days. TB infection in infants and children aged $<6$ years was assessed using the tuberculin skin test (TST, cut-off $\geqslant 5 \mathrm{~mm}$ induration), while adult contacts were screened with the QuantiFERON-TB GoldPlus test (QFT, Qiagen). All TST/QFT-positive individuals were presumed to be infected and underwent both chest radiography and a visit by a paediatrician, for children, or by a pulmonologist/ infectious disease specialist for adults. Seventeen individuals with presumed LTBI were diagnosed: five children (all $<5$ years of age) plus five close and seven occasional adult contacts. Subjects having LTBI received isoniazid $\left(10 \mathrm{mg} \cdot \mathrm{kg}^{-1} \cdot \mathrm{day}^{-1}\right)$ for 6 months. For every TST-negative child tested $<8$ weeks since the last contact, isoniazid syrup $10 \mathrm{mg} \cdot \mathrm{kg}^{-1} \cdot \mathrm{day}^{-1}$ for 2 months was proposed until TST was repeated. None of them eventually converted TST.

A total of seven subjects were diagnosed active TB during the screening process: four children $<5$ years (three with pulmonary and one with lymph node disease), and three close contact adults with pulmonary disease. Three out of the seven patients with active TB (two with pulmonary and one with lymph node disease) were 
culture-confirmed and all but one of them had no bacilli on the direct smear. Patients without culture confirmation were diagnosed according to established clinical-radiological criteria [12]. Whole-genome sequencing was performed on all culture-confirmed cases to assess the strains' clonality [13]. Core-genome multilocus sequence typing (MLST) analysis, performed by Ridom SeqShpere (Ridom Bioinformatics GmbH, Münster, Germany), showed that the strains were clustering with that of the index case (figure 1c). The index case (TS1) harboured a mixed population of the same clone and the predominant clone was transmitted to TS3 (infant) and TS7 (adult close contact), while the minority population was responsible for the disease in TS2 (infant).

All cases were treated with standard regimen for drug-susceptible TB as the Mycobacterium tuberculosis strain was pan-susceptible [13]. Epidemiological investigation revealed that all four active paediatric TB cases were vaccinated by the paediatrician within 45 days prior to the index case's diagnosis.

The frequency of active TB disease occurring during the likely highest period of infectiousness of the index case was different among adults and children. In fact, among the 123 occasional adult contacts (attending the wedding or the refresher course), seven had LTBI (5.69\%) and none had active disease.

In contrast, among the 737 children having a short contact with the index case in the 45 days preceding her diagnosis, five were found with LTBI $(0.67 \%)$ and four with active TB disease $(0.54 \%)$.

The estimated relative risk of developing active TB following an occasional contact with the index case was increased in children compared to adults (respectively $R=1.00545$ and $R=0.99457 ; 95 \%$ CI ratio of relative risk from 0.99248 to 1.00757 ).

To our knowledge this is the largest TB outbreak generated by a sputum smear-positive healthcare worker in an outpatient setting ever reported, and the first one comparing secondary cases among children and adults following an occasional contact.

In 2016, in the Trieste municipality (204234 inhabitants) active TB disease was diagnosed in 10 adults. No child $<6$ years of age was diagnosed with active TB disease in the previous 2 years. Therefore, a single healthcare facility outbreak contributed significantly to the epidemiology of paediatric TB in the region and provided three important lessons.

Firstly, when the infectiousness of the index cases is high, even an occasional contact $(<30 \mathrm{~min})$ may be sufficient to infect and subsequently evolve rapidly towards TB disease in vulnerable individuals such as children $<5$ years old. Therefore, the $8 \mathrm{~h}$ threshold used to identify contacts with risk of TB transmission needing further investigation may not be appropriate in all settings and should be revisited.

Secondly, our study provides evidence that vulnerable populations should be protected from the risk of developing active TB also in outpatient settings (as expected, children progress to TB disease at a higher rate than adults).

Finally, in Italy there is currently no obligation for outpatient healthcare workers (and paediatricians) to undergo regular screening for infectious diseases such as TB. In this case, characterised by a long patient delay, regular screening of outpatient healthcare workers could have prevented transmission in a number of individuals.

A thorough cost-effectiveness evaluation is needed to establish whether a national screening policy for outpatient healthcare workers (besides all healthcare workers) should be developed and implemented to protect the most vulnerable groups (including children $<5$ years of age).

Roberto Luzzati $\oplus^{1,10}$, Giovanni Battista Migliori $\oplus^{2,10}$, Matteo Zignol ${ }^{3,10}$, Daniela Maria Cirillo ${ }^{4,10}$, Massimo Maschio ${ }^{5}$, Riccardo Tominz ${ }^{6}$, Giovanna Ventura ${ }^{5}$, Valentino Patussi ${ }^{6}$, Lia D'Ambrosio $^{2,7}$, Rosella Centis ${ }^{2}$, Franco Michieletto ${ }^{6}$, Alberto Trovato $^{4}$, Francesco Salton ${ }^{8}$, Marina Busetti, Manuela Di Santolo ${ }^{9}$, Mario Raviglione ${ }^{3,10}$ and Marco Confalonieri ${ }^{8,10}$

${ }^{1}$ Unit of Infectious Diseases, Dept of Medical, Surgical and Health Sciences, University of Trieste, Trieste, Italy. ${ }^{2}$ WHO Collaborating Centre for TB and Lung Diseases, Maugeri Care and Research Institute, IRCCS, Tradate, Italy. ${ }^{3}$ Global TB Programme, World Health Organization, Geneva, Switzerland. ${ }^{4}$ Emerging Bacterial Pathogens Unit, Division of Immunology and Infectious Diseases, San Raffaele Scientific Institute, HSR, Milan, Italy. ${ }^{5}$ Institute for Maternal and Child Health, IRCCS "Burlo Garofolo", Trieste, Italy. ${ }^{6}$ Dept of Prevention, Local Health Authority and University of Trieste, Trieste, Italy. ${ }^{7}$ Public Health Consulting Group, Lugano, Switzerland. ${ }^{8}$ Pulmonology Unit, University Hospital of Cattinara, Trieste, Italy. ${ }^{9}$ Microbiology Unit, University Hospital ASUITS, Trieste, Italy. ${ }^{10}$ These authors contributed equally.

Correspondence: Marco Confalonieri, Pulmonology Unit, University Hospital of Cattinara, 34149 Trieste, Italy. E-mail: marco.confalonieri@asuits.sanita.fvg.it

Received: July 132017 | Accepted after revision: Aug 162017

First published online Nov 2, 2017; republished Nov 10, 2017 with amendments to the authors' affiliation details. 


\section{References}

1 Uplekar M, Weid D, Lönnroth K, et al. WHO’s new end TB strategy. Lancet 2015; 385: 1799-1801.

2 Lönnroth K, Migliori GB, Abubakar I, et al. Towards tuberculosis elimination: an action framework for low-incidence countries. Eur Respir J 2015; 45: 928-952.

3 Getahun H, Matteelli A, Abubakar I, et al. Management of latent Mycobacterium tuberculosis infection: WHO guidelines for low tuberculosis burden countries. Eur Respir J 2015; 46: 1563-1576.

4 Gillini L, Centis R, D'Ambrosio L, et al. Is Europe ready to reach tuberculosis elimination? An outbreak report from Southern Italy. Eur Respir J 2015; 46: 274-277.

5 Esposito S, D'Ambrosio L, Tadolini M, et al. ERS/WHO Tuberculosis consilium assistance with extensively drug-resistant tuberculosis management in a child: case study of compassionate delamanid use. Eur Respir J 2014; 44: 811-815.

6 Borgia P, Cambieri A, Chini F, et al. Suspected transmission of tuberculosis in a maternity ward from a smear-positive nurse: preliminary results of clinical evaluations and testing of neonates potentially exposed, Rome, Italy, 1 January to 28 July 2011. Euro Surveill 2011; 16: 19984.

7 Veen J. Microepidemics of tuberculosis: the stone-in-the-pond principle. Tuber Lung Dis 1992; 73: 73-76.

8 Communicable Diseases Network Australia. CDNA national guidelines for public health management of TB 2015. Canberra, Department of Health 2015. www.health.gov.au/internet/main/publishing.nsf/content/D140EDF48C0A0 CEACA257BF0001A3537/\$File/TB-2.0-april2015.pdf Date last accessed: August 4, 2017.

9 World Health Organization. Tuberculosis and air travel. Guidelines for prevention and control. 3nd Edn. WHO/ HTM/TB/2008.399. Geneva, World Health Organization, 2013.

10 Erkens CG, Kamphorst M, Abubakar I, et al. Tuberculosis contact investigation in low prevalence countries: a European consensus. Eur Respir J 2010; 36: 925-949.

11 European Centre for Disease Prevention and Control. Investigation and control of tuberculosis incidents affecting children in congregate settings. Stockholm, ECDC, 2013.

12 Migliori GB, Zellweger JP, Abubakar I, et al. European union standards for tuberculosis care. Eur Respir J 2012; 39: 807-819.

13 Kohl TA, Diel R, Harmsen D, et al. Whole-genome-based Mycobacterium tuberculosis surveillance: a standardized, portable, and expandable approach. J Clin Microbiol 2014; 52: 2479-2486. 